\title{
Technology and Application of Centralized DC Power Supply for LED Street Lamp
}

\author{
Rutong Zhang1, a, Xiaoshu Huang ${ }^{1}$, Bin Zhou', Yang Yang ${ }^{1}$ \\ ${ }^{1}$ NARI Technology Developmnet Co.,Ltd. Nanjing 211106,China \\ a zhangrutong@sgepri.sgcc.com.cn
}

Keywords: LED street lamp; centralized power supply; DC protection; power supply distance; Energy efficiency;

Abstract: With the continuous expansion of the scale of urban lighting, LED (light-emitting diode) lamps with a long life, high luminous efficiency, in many cities gradually popularized into the practical stage. In the traditional AC power system, the driving power of LED is the main failure point of LED lamps, and it has a huge maintenance cost. Aiming at this problem, this paper presents a scheme of DC power supply system for LED road lighting, and analyzes the key problems such as DC protection and power supply distance, and makes an energy efficiency comparison comparison with sodium lamp and AC power supply LED lamps. It is concluded that the DC power supply system is a reasonable choice for urban lighting.

\section{Introduction}

China's electricity consumption accounts for about $13 \%$ of the total electricity consumption, of which urban road lighting accounts for the entire lighting power consumption of $25 \%-30 \%$. Most of the traditional urban road lighting using high pressure sodium lamp, this lamp has a large power consumption, low efficiency, short life, repeated slow start, the system light efficiency, heat, color reproduction and other issues, the urgent need to develop more energy efficient and reliable Road lighting light source [1]. High light effect LED lamp in recent years at home and abroad rapid development of the new light source, with high luminous efficiency, no pollution, long life, vibration resistance, easy to damage, instantaneous start and so on. The use of LED lamp instead of the traditional high pressure sodium lamp, in ensuring the quality of the premise of lighting, you can save at least $50 \%$ of the electricity [2].

Traditional LED lamp with AC power supply, in the lamp part of the configuration AC/DC power supply LED lamp. AC/DC drive power for the low-power power supply, conversion efficiency is low, and the product short life of short electrolytic capacitors, high failure rate, resulting in LED lamp daily maintenance difficult, heavy workload, high cost. DC power supply LED street lamp system is a DC way to directly provide power to the LED lamp a program. In this program, in the street lamp control cabinet equipped with AC/DC power supply, the AC power conversion to DC $240 \mathrm{~V}$, and the distribution line output to the LED lamp; in the street with high reliability DC/DC drive power instead of the original AC/DC drive power supply. DC power supply centralized layout, which will help improve the efficiency of power conversion, and simplify the LED street lamp drive power, and DC power supply voltage stability, to avoid the impact of LED lamps, LED lamps will help improve the life of the lamp drive power supply life and reliability, Maintenance cycle, reduce maintenance costs; AC and DC power conversion part of the DC installed in the road control cabinet, to avoid the maintenance of the pole, is conducive to reducing maintenance workload [3]. 


\section{Design of DC power supply system for LED street lamp}

LED street light centralized DC power supply system consists of street lamp monitoring and management system, street lights integrated distribution cabinet, single lamp drive controller, LED lamp module, street lamp poles, power supply lines, transformers and other equipment. The overall block diagram of the DC power supply system is shown in Figure 1.

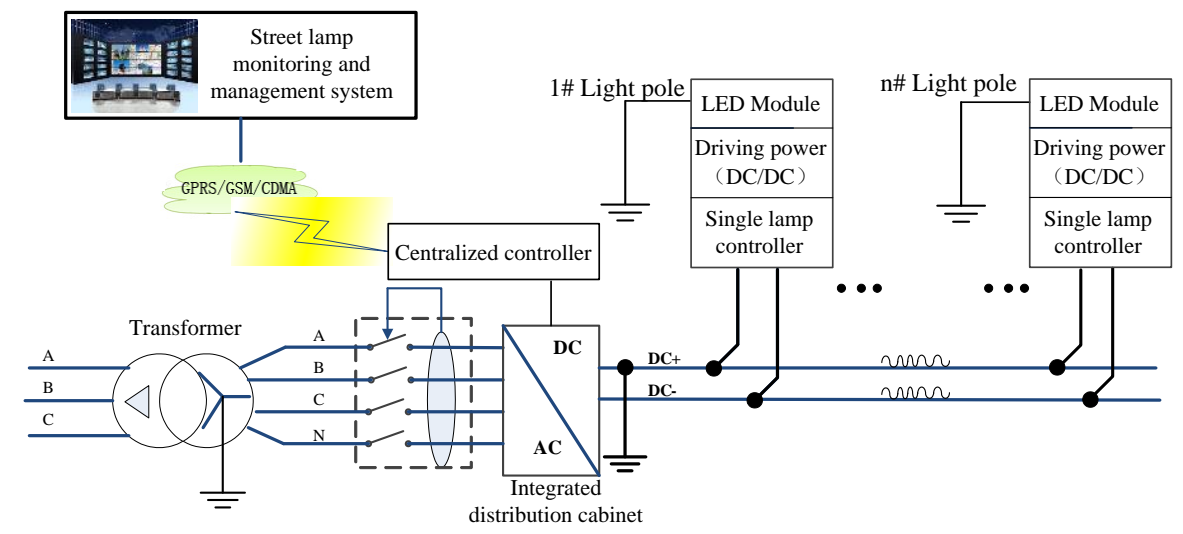

Fig. 1 Integral block diagram of DC power supply system for LED street lamp

Transformers mainly play the role of AC isolation, therefore, LED driver can use non-isolation program, to avoid the optocoupler, transformer and other isolation components caused by instability in the system reliability. Single lamp drive controller includes a single lamp driver and a single lamp controller to complete the LED module drive, single lamp power measurement, status monitoring and communication functions. The integrated distribution cabinet includes AC/DC rectifier module, centralized controller, leakage protector, lightning protection device, DC circuit breaker and other modules. The integrated power distribution cabinet mainly completes the DC power supply and safety protection function of the street lamp. LED lamp provide 240V DC. Where in the centralized controller comprises an electric power carrier communication module and a GPRS communication module. The power carrier communication module communicates with the single lamp drive controller, collects the LED parameter information of the LED street lamp, and monitors the working status of the LED street lamp. GPRS communication module is responsible for the integrated power distribution cabinet within the scope of the collection of street light information remote transmission to the street management center, according to the needs of automated management, LED lamp can be realized automatic operation control and management.

As the power supply line naturally exists to the ground distributed capacitance, AC current through this capacitor leakage current, and the distribution of capacitance will be line length, insulation conditions, weather conditions, soil moisture and other changes in the external situation, resulting in leakage current with the environment There is a big change, resulting in leakage protection setting difficult. If the value is too small, rainy days easy to move; if the value is too large, the occurrence of electric shock when people fail to protect the value of protection to protect the switch is easy to move, and can't achieve the safety of personnel protection. The use of DC will not be formed by the line capacitance of the leakage current leakage, which excludes the impact of line leakage current, according to the requirements of personal electric shock protection set accurate leakage protection settings, to achieve personal safety protection.

The body's ability to withstand electric shock is much smaller than the tolerance of DC electric shock, which also improves the DC power supply on the safety of electric shock capacity. According to the "GB/T 13870.1-2008 current on the human and livestock effect", the human body tolerance to the current limit is $30 \mathrm{~mA}$, while the human tolerance to DC current limit is $100 \mathrm{~mA}$; 
IEC standard " IEC 60884-2-4-2007 Plugs and socket-outlets for household and similar purposes "also clearly stipulates that the upper limit of the AC voltage is AC $50 \mathrm{~V}$ and the upper limit of the direct current (no ripple) voltage is DC120 volts, and there is a significant difference between the two.

DC bus protection on the DC bus, a total of four parameters can be set, respectively, leakage protection settings, leakage protection delay, overload protection settings, overload protection delay. Overload protection delay refers to the detection of current exceeds the set value, the delay action, usually branch set to 0 delay, bus set a delay, so that when the branch overload fault occurs, the branch first trip, cut off the fault, bus Protection does not move; if the slip protector fails, no trip cut fault, the delay after the bus protection action [4].

To Street Lamps, the same section of the lamps are generally the same model, and the light pole spacing for the same spacing. As shown in Figure 2, set the rated current of the lamp to I, the distance between the poles is 1 , the interval circuit resistance $R$, there are $n$ pole. It is easy to get the last pen pole interval on the cable current 2I, the first light pole interval cable current nI.

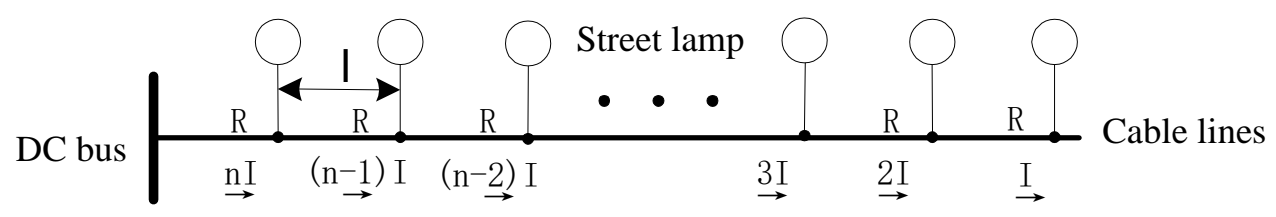

Fig. 2 Schematic diagram of DC power supply circuit for LED street lamp

According to $U=I R$, it can be concluded that the total voltage drop $\left(U_{\mathrm{wd}}\right)$ of the circuit is the sum of the voltage drop across all poles:

$$
\begin{aligned}
& U_{\mathrm{vd}}=\mathrm{nI} \times \mathrm{R}+(\mathrm{n}-1) \mathrm{I} \times \mathrm{R}+\cdots+\mathrm{I} \times \mathrm{R} \\
& U_{\mathrm{vd}}=\frac{n(n+1)}{2} \times I \times \mathrm{R} \\
& \mathrm{R}=\mathrm{n} \times l=\sqrt{\frac{2 \times 1 \times U_{v d}}{\frac{P}{U_{0}} \times \rho \times 10^{-s}}} \\
& \mathrm{R}=\mathrm{K} \times \sqrt{U_{\mathrm{vd}}}
\end{aligned}
$$

In the formula $3, \mathrm{U}_{\mathrm{vd}}$ is the voltage drop between the head and the end of the street lamp power supply line; $\rho$ is the line resistivity; $\mathrm{P}$ is the integrated power of the LED lamp; $\mathrm{U}_{0}$ is the terminal voltage of the LED lamp; the other is constant, Distance $R$ and $\sqrt{U_{v d}}$ can be seen as a proportional relationship, for the selected road conditions $\mathrm{K}$ is constant.

\section{System implementation}

DC power supply LED street lamp power supply system generally by the street integrated control cabinet (including rectifier, etc.), LED lamp (including single lamp drive controller), power lines, the main station system and other components. LED street lamp centralized DC power supply system structure shown in figure 3. 


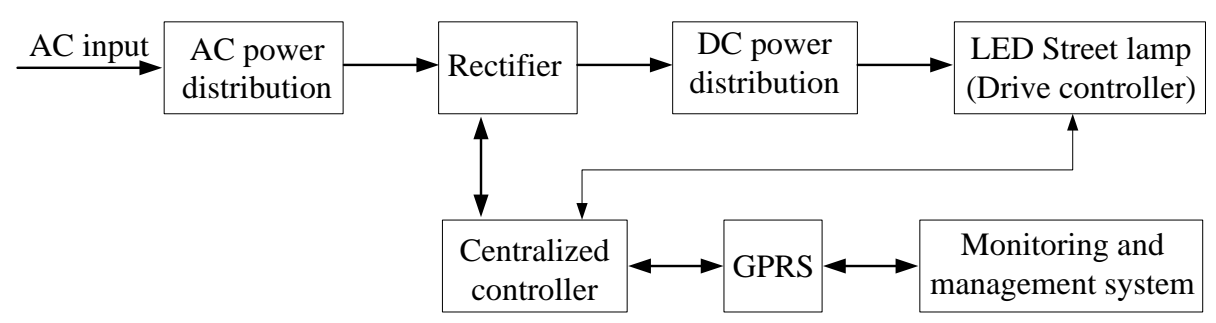

Fig. 3 Structure diagram of LED street lamp centralized DC power supply system

The integrated control cabinet of each LED integrates the AC/DC drive power of each LED in the control cabinet, improves the power conversion efficiency and provides the stable rectifier module power supply with $\mathrm{N}+1$ thermal redundant backup. DC conversion, communication and control, power distribution and protection functions. AC and DC conversion for the LED lamp to provide DC power supply, the output power of the entire cabinet can be adjusted according to actual needs; communication and control is the streetlight control center and the communication between the lights, to achieve street lamp control, status detection and other data upload and Power distribution and protection, including short circuit, overload, leakage, lightning protection, security and other protection functions; in practical applications, the general integrated control cabinet can control more than 100 LED lamps.

The single lamp drive controller converts the high DC voltage to the low DC voltage required for the LED fixture and is connected between the DC output of the control cabinet power module and the LED module. The single-lamp drive controller is not affected by the EMC effect of the AC grid on the lighting of the fixture and is not affected by the lightning transmission from the AC grid side (it is also necessary to resist the impact of the lightning strike on the DC link) without the need for EMC, rectification, Factor correction, filtering, power drive, inverter buck circuit and components [5]. Simple constant pressure type only very few auxiliary components, direct output to the series of LED light source, or constant current circuit output constant current to the series of LED light source better. In this way, the circuit of the lamp control device is very simple, the reliability is very high, the life can be very long, which is very favorable for the use of poor environmental conditions and tunnel lighting works.

At present, most of the street lamp power supply cable with three-phase four-wire $(\mathrm{A} / \mathrm{B} / \mathrm{C} / \mathrm{N})$ or three-phase five-wire $(\mathrm{A} / \mathrm{B} / \mathrm{C} / \mathrm{N} / \mathrm{PE})$ to the bottom of the poles, The way for the lighting to provide single-phase AC power supply, cable diameter is generally $16 \mathrm{~mm} 2-25 \mathrm{~mm} 2$. If the DC power supply to transform the line, the line output of the DC power supply, in order to reduce the construction workload and construction difficulty, can be $\mathrm{A} / \mathrm{B} / \mathrm{C}$ three lines in the output shorted, as one of the DC power supply Pole, $\mathrm{N}$ line as the other pole of the DC power supply, to ensure that the transmission to the lamps are the same group of DC power supply of the two poles. The grounding mode can't be changed.

In the use of DC centralized power supply on the basis of the configuration of urban lighting intelligent monitoring system, can achieve urban lighting management information, intelligent [6]. Urban lighting intelligent monitoring system mainly by the support layer, data layer, application layer and presentation layer. The system layer mainly includes the urban lighting operation management system and related business integration, is the system control center. Communication layer uses a variety of ways to achieve system communication, to meet the needs of running lights at the same time, for the value-added services to provide reserved control. The equipment layer consists of street lighting integrated control cabinet (power supply, control, communication), single lamp controller, street lamp, landscape lamp, light pole, wire and other equipment. The main station system and the streetlight centralized controller are transmitted via GPRS. 


\section{Energy efficiency evaluation}

Energy-saving and emission reduction benefits mainly include the reduction of coal combustion and the reduction of harmful gases such as carbon dioxide and sulfur dioxide. According to the data released by the National Bureau of Statistics, by 2015 the number of urban street lights is about 30 million, according to street lamp energy-saving engineering experience, generally with the original high-pressure sodium lamp power of $40 \%$ can achieve the same lighting effect [7]. The current common scheme is to replace the existing $250 \mathrm{~W}$ high-pressure sodium lamp with a $100 \mathrm{~W}$ LED street lamp. The power consumption of the high-pressure sodium lamp rectifier is $70 \mathrm{~W}$, the efficiency of the AC/DC rectifier module is $88 \%$, the efficiency of the DC/DC drive module is $97 \%$, The integrated control cabinet power supply efficiency of 94\%; AC line power factor of 0.85, street lamp spacing is assumed to be 30 meters, the cable selection YJV22 $4 \times 25$ cable, the resistivity of $0.727 \Omega / \mathrm{km}$, the following 100 lights, The length of $3 \mathrm{~km}$ as an example of the calculation, the parameters shown in Table 1.

Tab.1 Basic parameter table

\begin{tabular}{|c|c|c|c|}
\hline Category & HPS & LED street lamp (AC) & LED street lamp (DC) \\
\hline Source power (W) & 250 & 100 & 100 \\
\hline Input power (W) & 320 & 114 & 103 \\
\hline Number of lamps & 100 & 100 & 100 \\
\hline Line voltage (V) & 220 & 220 & 240 \\
\hline
\end{tabular}

AC power supply system and centralized DC power supply system line loss formula as shown in Equation 5 and 6 . Where $\Delta \mathrm{P}_{\mathrm{AC}}$ is the AC power supply line loss power; $\Delta \mathrm{P}_{\mathrm{DC}}$ is the DC power supply line loss power, $\mathrm{n}$ is the number of lights on the same circuit; $\mathrm{P}$ is the single lamp integrated power; $\mathrm{U}_{0}$ is the starting voltage; $\cos \varphi$ is the line power factor; 1 is the street light spacing; pis the street lamp resistivity.

$$
\begin{aligned}
& \Delta P_{A C}=6 \times\left[\frac{n(n+1)}{2}\right] \times\left(\frac{p}{U_{0} \cos \varphi}\right)^{2} \times 3 l_{\rho} \\
& \Delta P_{D C}=4 \times\left[\frac{n(n+1)}{2}\right] \times\left(\frac{P}{U_{0}}\right)^{2} \times 2 l_{\rho}
\end{aligned}
$$

From the formula 5 and 6 can be calculated in the AC power supply under the high pressure sodium lamp and LED street lamp loss and centralized DC power supply LED street lamp line loss. It can be seen from the figure that LED lamp greatly reduce the line loss. And in the centralized DC power supply under the LED street lamp line loss is only equivalent to AC power supply under half of the LED lamp, so concentrated DC power supply on the loss of energy-saving effect is obvious.

At present, China's thermal power plant coal consumption of $326 \mathrm{~g} / \mathrm{kWh}, \mathrm{CO}_{2}$ emissions $997 \mathrm{~g} / \mathrm{kWh}$ estimates, assuming that half of the country's street lamps to implement centralized DC LED lamp energy-saving transformation, the phase of the coal-fired power generation accounts for about $65 \%$ Than the high-pressure sodium lamp can reduce the annual $\mathrm{CO}_{2}$ emissions of about 9.4443 million tons, saving about 308.94 million tons of coal, compared to the exchange of LED lamp per year can reduce $\mathrm{CO}_{2}$ emissions of about 190,700 tons, saving about 62,400 tons of coal, Showing its energy-saving emission reduction benefits significantly.

\section{Conclusions}

DC power supply LED street light system and AC power supply LED street lamp system, has obvious advantages. DC power supply, LED lighting with DC/DC drive power supply, power supply structure is simplified, more efficient, to avoid the use of filter electrolytic capacitors, thereby extending the life of LED lamp; AC and DC power conversion part of the road installed in the DC Control cabinet, to avoid the maintenance of the poles, is conducive to reducing 
maintenance workload, and in the actual operation of more energy efficient and economic; the same time, DC lines can be set to improve the protection and has a far distance from the power supply. In addition, DC distribution network and the new energy storage, electric vehicle charging pile, wifi hot spots, video surveillance and other comprehensive integration [8], intelligent lighting system configuration, is conducive to urban management from passive management to active service change, from manual management to Automation management change, improve the level of automation of urban management, for the wisdom of the city, safe city to provide support.

\section{References}

[1] Rongnan Ye. LED street lights for urban road lighting [J]. Lighting Engineering, 2009, 20 (Z1)

[2] Yihua Weng. Application Analysis and Prospect of High Pressure Sodium Lamp and LED in Urban Road Lighting [J]. China Lighting \& Electric Appliance, 2012, (4): 17-21.

[3] Celidonio M, Fionda E, Pulcini L, et al. A Centralised DC Power Supply solution for LED lighting networks[C]//Energy Conference (ENERGYCON), 2014 IEEE International. IEEE

[4] Wenliang Zhang, Yong Tang, Nanchao Zeng. Application of multi-terminal HVDC technology and its application prospect [J]. Power System Technology, 2010, 34 (9): 1-6.

[5] Weixing Lin, Jinyu Wen, Shijie Cheng. DC-DC autotransformer and its extension technology[J]. South China Power Grid Technology, 2016 (1).

[6] Zhaobei Shi, Yushuang Wang, Dengjun Yan. Status and Development Trend of Energy Saving Technology for Urban Street Lighting [J]. Chinese Journal of Lighting, 2009 (10)

[7] Baojun Li, Daming Mou, Yiliang Yao, et al. Comparative analysis of energy efficiency of LED street lamp and high pressure sodium lamp [J] .Journal of Illuminating Engineering, 2012

[8] Yuhong Xie. DC microgrid monitoring and protection system research [D]. Southwest Jiaotong University, 2014. 